Draft Version November 17, 2018

Preprint typeset using $\mathrm{LAT}_{\mathrm{E}} \mathrm{X}$ style emulateapj v. 5/2/11

\title{
ON THE GALACTIC SPIN OF BARRED DISK GALAXIES
}

\author{
Bernardo Cervantes-Sodi ${ }^{1}$, Cheng Li ${ }^{1}$, Changbom Park ${ }^{2}$ And Lixin Wang ${ }^{1}$ \\ Draft version November 17, 2018
}

\begin{abstract}
We present a study of the connection between the galactic spin parameter $\lambda_{d}$ and the bar fraction in a volume-limited sample of 10,674 disk galaxies drawn from the Sloan Digital Sky Survey Data Release 7 . The galaxies in our sample are visually classified into galaxies hosting long or short bars, and non-barred galaxies. We find that the spin distributions of these three classes are statistically different, with galaxies hosting long bars with the lowest $\lambda_{d}$ values, followed by non-barred galaxies, while galaxies with short bars present typically high spin parameters. The bar fraction presents its maximum at low to intermediate $\lambda_{d}$ values for the case of long bars, while the maximum for short bars is at high $\lambda_{d}$. This bi-modality is in good agreement with previous studies finding longer bars hosted by luminous, massive, red galaxies with low content of cold gas, while short bars are found in low luminosity, low mass, blue galaxies, usually gas rich. In addition, the rise and fall of the bar fraction as a function of $\lambda_{d}$, within the long-bar sample, shown in our results, can be explained as a result of two competing factors: the self-gravity of the disk that enhances bar instabilities, and the support by random motions instead of ordered rotational motion, that prevents the formation/growth of bars.

Subject headings: galaxies: fundamental parameters - galaxies: general - galaxies: structure - galaxies : spiral - galaxies : statistic
\end{abstract}

\section{INTRODUCTION}

If we take a look of an image of a galaxy such as NGC 1300, a special feature that immediately catches our attention is the prominence of the bar. At the same time, we can find galaxies of similar mass and overall morphology with no sign of bar presence. The presence of such prominent non-axisymmetric features must certainly shape the structure and secular evolution of the host galaxies by exerting tidal torques which lead to a redistribution of the galactic constituents (Friedli \& Benz 1993).

Through analytical and numerical calculations previous studies have shown that bars produce a redistribution of mass and angular momentum in the galactic disk (Hohl 1971; Weinberg 1985; Debattista \& Sellwood 2000; Athanassoula 2002; Martinez-Valpuesta et al. 2006; Hwang et al. 2013). Stellar density waves can be driven by galactic bars leading to the formation of spiral arms (Lindblad 1960; Toomre 1960; Elmegreen \& Elmegreen 1985) and ring structures (Schwartz 1981; Buta \& Combes 1996). Bars can also funnel material toward the galaxy center; this is specially the case for the collisional gas component that can dissipate energy during shocks and flow inwards (Shlosman, Frank \& Begelman 1989; Friedli \& Benz 1993). This inflow will produce an accumulation of material in the central region that can help in the build-up of disk-like bulges or pseudo-bulges (Sheth et al. 2005; Laurikainen et al. 2007; Okamoto 2012), most prominently in the case of early-type galaxies where bars appear larger (Elmegreen \& Elmegreen 1985, 1989; Erwin 2005). As a result of these gas in-

\footnotetext{
bernardo@shao.ac.cn

${ }^{1}$ Partner Group of the Max Planck Institute for Astrophysics and Key Laboratory for Research in Galaxies and Cosmology of Chinese Academy of Sciences, Shanghai Astronomical Observatory, Nandan Road 80, Shanghai 200030, China

2 Korea Institute for Advanced Study, Dongdaemun-gu, Seoul 130-722, Republic of Korea
}

flows, higher molecular gas concentrations are found in the central regions of barred galaxies (Sakamoto et al. 1999; Sheth et al. 2005), as well as younger populations in the bulges of barred galaxies when compared with unbarred ones (Coelho \& Gadotti 2011). Numerical simulations by Friedli, Benz \& Kennicutt (1994) show not only inflow, but also outflow induced by the bar, that can facilitate chemical mixing and trigger star formation as found in observational studies (Hummel et al. 1990; Huang et al. 1996; Ellison et al. 2011).

If bars funnel gas inwards and most of massive galaxies host super-massive black holes (SMBHs), a natural consequence would be an enhanced active galactic nucleus (AGN) activity. Mechanisms such as "bars within bars" (Shlosman et al. 1989, 2000) and nuclear spirals (Martini \& Pogge 1999; Márquez et al. 2000) have been suggested to transport gas to the vicinity of the SMBH, but in this respect no consensus has been reached, with some authors proposing the presence of bars as a triggering factor for AGN activity (Knapen et al. 2000; Laine et al. 2002; Laurikainen et al. 2004), while others are more sceptical (Moles et al. 1995; Mulchaey \& Regan 1997; Lee et al. 2012b; Oh et al. 2012).

Some numerical simulations (Hasan \& Norman 1990; Norman, Sellwood \& Hasan 1996; Athanassoula et al. 2005) show weakening of the bars, and in some cases dissolution, by a central mass concentration (CMC) or gravity torques (Bournaud et al. 2005). In order to explain the large proportion of barred galaxies observed in different observational surveys, mechanisms such as the dissolution and reformation of bars as recurrent transient structures has been proposed (e.g. Combes 2000; Bournaud \& Combes 2002), as well as the survival of bars to the presence of a CMC (Shen \& Sellwood 2004; Debattista et al. 2006)

Recently, the relevance of bars in galaxy evolution has motivated a large number of studies. The fraction of 
barred galaxies found in optical images varies depending on the method used to identify galaxies with bars and on sample selection, with the typical fraction between $30 \%$ up to 50\% (Barazza et al. 2008; Aguerri et al. 2009; Nair \& Abraham 2010; Lee et al. 2012a, henceforth Lee+12). The fraction appears even higher when near-infrared images are used, where weak bars are not obscured by dust and hence are more easily identified (Marinova \& Jogee 2007; Menéndez-Delmestre et al. 2007), appearing at NIR wavelengths as strong bars (Buta et al. 2010). Analyses of the bar fraction in the Hubble Deep Fields found a dramatic drop of barred systems at $z>5$ (Abraham et al. 1999; van den Bergh et al. 2002) later confirmed by Sheth et al. (2008) studying a sample of luminous face-on spirals from the $2 \mathrm{deg}^{2}$ Cosmic Evolution Survey, although results from others studies report the contrary, a constant bar fraction since $z \leq 1$ (e. g. Elmegreen et al. 2004; Jogee et al. 2004). Sheth et al. (2008) also find that the bar fraction in spiral galaxies is a strong function of stellar mass, color and bulge prominence such as it increases for more massive, luminous redder systems with a slight preference for bulge-dominated galaxies. Using a smaller sample of 190 galaxies from the Coma Cluster, Méndez-Abreu et al. (2010) found that bars are hosted by galaxies in a tight range of luminosities $\left(-20 \lesssim M_{r} \lesssim\right.$ -17) and masses $\left(10^{9} \lesssim M_{*} / M_{\odot} \lesssim 10^{11}\right)$.

Nair \& Abraham (2010) also report this strong dependence on mass, but given that their sample contain low mass galaxies, they are able to assess that the bar fraction is bimodal on mass, with a high fraction at the low mass end, mostly on late-type spirals, a decrease of the fraction at intermediate mass galaxies $\left(\log \left(M / M_{\odot} \sim 10.2\right)\right.$, and increasing again at the high mass end, this time most of the barred galaxies being early-type spirals. Recently Lee+12 found that the bar fraction strongly depends on color, with the fraction increasing significantly as the color becomes redder for the case of long bars, while the opposite is found for galaxies hosting short bars (see also Nair \& Abraham 2010; Masters et al. 2011). Regarding the dimensions of bars, Erwin (2005) found that early-type disk galaxies host bars a factor of $\sim 2.5$ longer than late-type disks, and Gadotti (2011) shows that longer bars tend to be hosted by galaxies with prominent bulges. In general, bars are found to be larger and stronger in early-type galaxies (Elmegreen \& Elmegreen 1985, 1989; Aguerri et al. 2009; Hoyle et al. 2011).

All these evidence leads to conclude that bars play a major role in the secular evolution of galaxies (e. g. Kormendy \& Kennicutt 2004; Laurikainen et al. 2007) and can be sensitive to dynamical interactions of the host galaxy with its neighbors (Lee+12). On previous works, some of us have investigated the role of the galactic spin on shaping the structure and morphology of present day galaxies. Features such as galaxy color, the thickness of the disk, bulge prominence, metallicity and AGN activity are strongly dependent on the spin of the systems (e. g. Hernandez \& Cervantes-Sodi 2006; Cervantes-Sodi \& Hernandez 2009; Cervantes-Sodi et al. 2011). Numerical studies (Efstathiou, Lake \& Negroponte 1982, henceforth ELN; Mayer \& Wadsley 2004; Governato et al. 2009; Foyle, Courteau \& Thacker 2008) have shown that haloes with lower spin parameter, more compact and self-gravitating, are more prone to global instabilities and the formation of bars. Syer, Mao \& Mo (1999) tried to prove this hypothesis using a sample of $\sim 2,500$ galaxies selected randomly from the the ESO-Uppsala catalogue (Lauberts 1982), but could not find any trend pointing to barred galaxies having systematically lower spin. It must be taken into account that some systematics could be affecting the result, such that the majority are relatively late types (mainly $\mathrm{Sb}-\mathrm{Sd}$ types).

Later on, Athanassoula (2008) pointed out that the ENL stability criterion was over-simplistic and could not distinguish bar stable from bar unstable disks, specially because it does not take into account the disk velocity dispersion. On a previous study, Athanassoula \& Sellwood (1986) showed that velocity dispersion has an important influence on bar stability. In their studies they presented a disk with no halo, which was stable because of its high-velocity dispersion.

In the present study we want to address this topic, we will investigate further if the spin parameter has any direct intervention on the presence of bars in disk galaxies. The format of the paper is as follows. In Section 2 we describe the model to infer the spin parameter for disk galaxies in our sample. The sample is described in Section 3. In Section 4, we present our general results of the dependence of the bar fraction on the galactic spin. Discussion regarding our results is presented in Section 5 , followed by our conclusions in Section 6 .

\section{ESTIMATION OF THE GALACTIC SPIN PARAMETER}

To account for the spin of disk galaxies we use the $\lambda$ spin parameter as defined by Peebles (1971),

$$
\lambda=\frac{L|E|^{1 / 2}}{G M^{5 / 2}},
$$

where $E, M$ and $L$ are the total energy, mass and angular momentum of the configuration, respectively. In order to estimate this parameter for the galaxies in our sample, we adopt the model by Mo, Mao \& White (1998) as we did in Hernandez \& Cervantes-Sodi (2006). Here we briefly recall the main ingredients of the model. In the framework of the CDM scenario for galaxy formation, primordial density fluctuations give rise to haloes of dark matter of mass $M$, within which gas condenses and forms rotationally supported disks of maximum circular velocity $V_{d}$. The disk is expected to be thin and presenting an exponential surface density profile

$$
\Sigma(r)=\Sigma_{0} e^{-r / R_{d}}
$$

with $\Sigma_{0}$ the central surface density, $R_{d}$ the disk scalelength, and corresponding mass

$$
M_{d}=2 \pi \Sigma_{0} R_{d}^{2}
$$

which is a fraction $f_{d}$ of the halo mass

$$
M_{d}=f_{d} M
$$

If we describe the dark matter halo by a truncated singular isothermal sphere of radius $R_{H}=M G / V_{C}^{2}, \lambda=$ $\lambda^{\prime}$, with 


$$
\lambda^{\prime}=\frac{L}{\sqrt{2} M V_{c} R_{H}},
$$

as defined by Bullock et al. (2001). The dark matter halo is responsible for establishing a rigorously flat rotation curve along the disk, from where the angular momentum of the disk is

$$
L_{d}=2 M_{d} V_{c} R_{d}
$$

Assuming that the specific angular momentum of the disk is a fraction $j_{d}$ of that of the halo, we can express equation (5) as:

$$
\lambda=\left(\frac{\sqrt{2}}{G}\right)\left(\frac{f_{d}}{j_{d}}\right) R_{d} V_{d}^{2} M_{d}^{-1}
$$

We define $\lambda_{d}$ as the product $\lambda_{d}=\lambda j_{d}$. In the case of both components; baryons and dark matter, having the same specific angular momentum, $\lambda_{d}$ becomes $\lambda$ and we recover the expression we have used to assess the spin of disk galaxies in previous works i.e. Hernandez \& Cervantes-Sodi (2006) and Cervantes-Sodi et al. (2012). The input parameters in equation (7) to estimate the spin of disk galaxies are the disk scalelenght $R_{d}$, the disk mass $M_{d}$, the circular velocity $V_{d}$ and the disk mass fraction $f_{d} . R_{d}$ is taken from the SDSS $i$-band. As an estimation for the disk mass we use the stellar mass obtained from the MPA/JHU DR7 VAGC, which are based on fits to the SDSS five-band with the model of Bruzual \& Charlot (2003, see also Kauffmann et al. 2003). Given that the location of a galaxy in the Tully-Fisher (TF) relation does not depend on barredness (Courteau et al. 2003; Sheth et al. 2012), we determine confidently $V_{d}$ by the $r$-band Tully-Fisher relation from Pizagno et al. (2007) for all the late-type galaxies of the sample. Finally, following Gnedin et al. (2007), we derive the disk mass fraction in terms of the stellar surface density using:

$$
f_{d}=f_{0}\left(\frac{M_{*} R_{d}^{-2}}{10^{9.2} M_{\odot} k p c^{-2}}\right)^{p},
$$

where $p=0.2$, and $f_{0}$ is chosen using the Milky Way as a representative example. In Cervantes-Sodi et al. (2008) we proved the accuracy in our estimation of $\lambda$ by comparing the estimation using Equation 7 to values arising from numerical simulations of different authors. The result was a one-to-one correlation with small dispersion and no bias, leading to typical errors of less than $30 \%$. It has also been used by other groups finding it appropriate for different kind of studies (e.g. Puech et al. 2007; Gogarten et al. 2010; Muñoz-Mateos et al. 2011).

One would expect that galaxies with low spin parameters, being more compact and self-gravitating, be more prone to global instabilities. A simple criterion for the instability of a thin exponential stellar disk was proposed by ELN using a set of N-body experiments; namely, bar instability occurs if

$$
\epsilon_{c} \equiv \frac{V_{d}}{\left(G M_{d} / R_{d}\right)^{1 / 2}}<1.1
$$

which is a measure of the self-gravity of the disk. This value is to be compared to a self-gravitation exponential disk, with a value $\epsilon_{c}=0.63$. By combining equations (7) and (9), we get that

$$
\epsilon_{c}^{2}=\frac{\lambda_{d}}{\sqrt{2} f_{d}} .
$$

In this context we expect to find an increase of the fraction of barred galaxies as we move from high to low spinning galaxies.

\section{DATA}

The sample of galaxies analysed in this work comes from a previous study by Lee+12. It is a volume limited sample of galaxies extracted from the SDSS DR7 (Abazajian et al. 2009) with absolute magnitude in the $r$-band brighter than $M_{r} \leq-19.5+5 \log h$ within the redshift range $0.02 \leq z \leq 0.05489$. A total of 33,391 galaxies are identified from the Korea Institute for Advanced Study Value-Added Galaxy Catalogue (Choi et al. 2010). Given that we will focus our analysis on latetype galaxies, we divide this main sample into early- and late-type galaxies using the prescription by Park \& Choi (2005), where galaxies are segregated according to their morphology in the color versus color gradient and concentration index space. An additional visual inspection was performed to improve the accuracy of the morphology classification.

Galaxies are classified by visual inspection of $g+r+$ $i$ combined color images into long-barred, short-barred and non-barred systems. A galaxy having a bar that is larger than one quarter of the size of its host galaxy is classified as long-barred, while the opposite case is a short bar. The visual classification is more robust for face-on galaxies, where we also avoid internal extinction effects that would provide an underestimation of $V_{d}$ when using our TF relation. To this effect we limit our sample to galaxies with the minor-to-major axis ratio $b / a>0.6$. Our final sample contains 10,674 late-type galaxies, 3,240 with long bars and 698 with short bars. As described in Lee ++12 , the classification shows a good agreement with the classification performed by Nair \& Abraham (2010) considering the galaxies in common in the two samples, with our sample showing only a relatively small fraction of galaxies with short bars when compared with theirs due to a slightly stricter criterion. Figure 3 of Lee ++12 also shows some examples of late-type galaxy images with long and short bars.

For a more detailed description of the sample, and comparisons of the classification with previous works, we refer the reader to Lee+12.

\section{RESULTS}

\subsection{Dependence of the bar fraction on the galactic spin}

Using our $\lambda_{d}$ estimate from equation 17, we calculate the spin parameter for all the galaxies in our sample and look for the empirical distributions $P\left(\lambda_{d}\right)$ of each of the subsamples divided according to their morphology.

Figure 17 shows the spin distribution of disk galaxies in the sample divided in non-barred galaxies, barred ones, and those hosting long and weak bars. Theoretical (Shaw et al. 2006) and empirical (Hernandez et al. 2007) distributions of $\lambda_{d}$ are traditionally described by a log-normal function of the form 

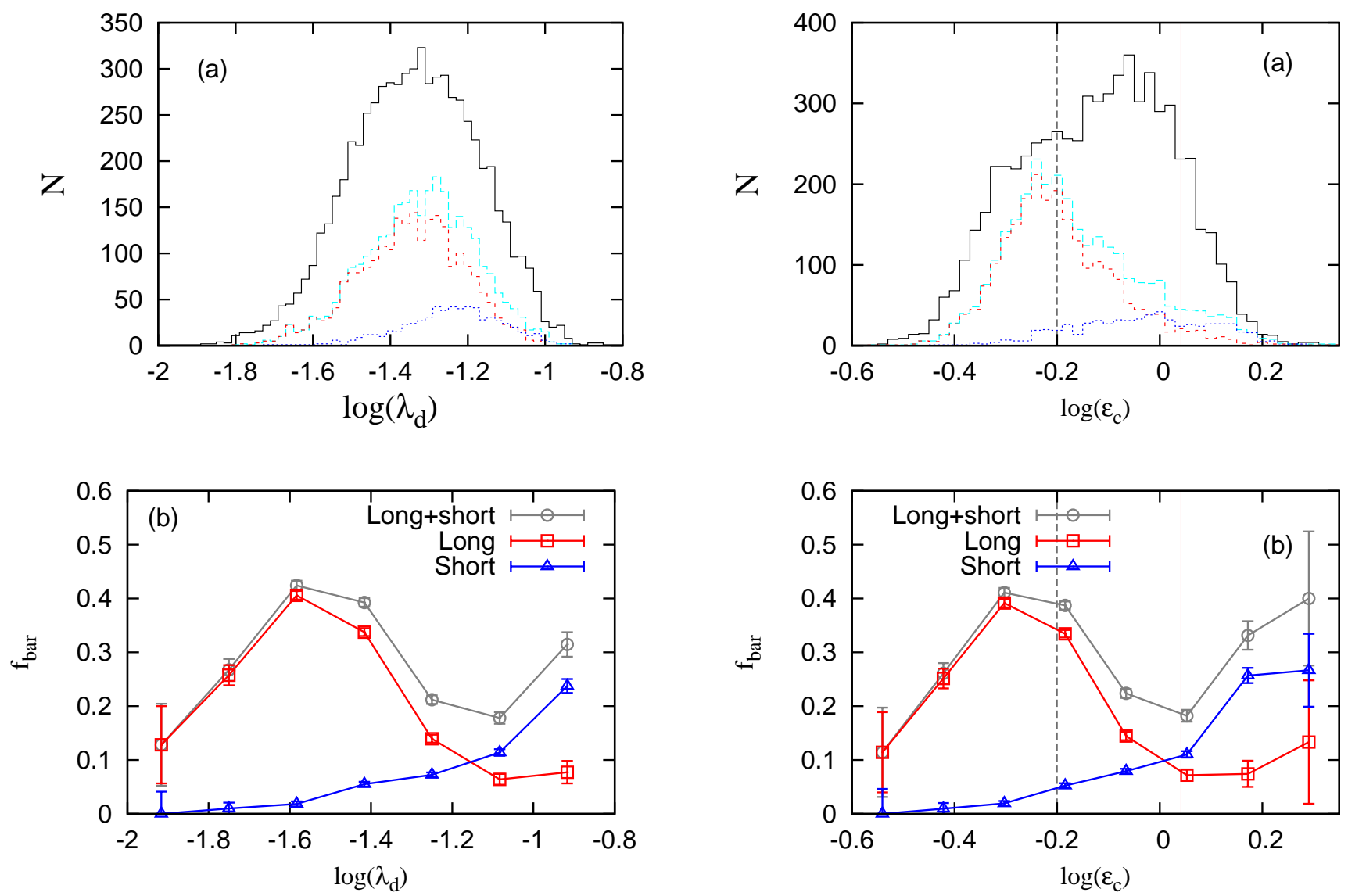

Fig. 1.- (a):histograms of $\lambda_{d}$ for non-barred galaxies (solid black line), barred galaxies (long plus short bars,long dashed cyan line), galaxies hosting long (short dashed red line) and short (dotted blue line) bars. (b): bar fraction as a function of $\lambda_{d}$ for long, short and long plus short barred galaxies.

TABLE 1

$\mathrm{P}\left(\lambda_{d}\right)$ Distributions.

\begin{tabular}{ccc}
\hline Sub-sample & $\lambda_{d_{0}}$ & $\sigma_{\lambda_{d}}$ \\
\hline Non-barred & 0.045 & 0.545 \\
Barred & 0.039 & 0.507 \\
Long & 0.035 & 0.449 \\
Short & 0.061 & 0.419 \\
\hline
\end{tabular}

$P\left(\lambda_{d_{0}}, \sigma_{\lambda_{d}} ; \lambda_{d}\right) d \lambda_{d}=\frac{1}{\sigma_{\lambda_{d}} \sqrt{2 \pi}} \exp \left[-\frac{\ln ^{2}\left(\lambda_{d} / \lambda_{d_{0}}\right)}{2 \sigma_{\lambda_{d}}^{2}}\right] \frac{d \lambda_{d}}{\lambda_{d}}$

The parameters describing the different $\lambda_{d}$ distributions on Figure 1a are listed on Table 1. The four distributions are statistically drawn form different underlying distributions confirmed by Kolmogorov-Smirnov tests, with a significance level $>99 \%$ for all cases. This shows that long bars are preferentially found in low spinning galaxies, while short barred galaxies present high spin when compared with non-barred galaxies. In Figure 10 we present the bar fraction as a function of $\lambda_{d}$, where we can clearly see two peaks, one at intermediate-low $\lambda_{d}$

FIG. 2.- (a):histograms of $\epsilon_{c}$ for non-barred galaxies (solid black line), long plus short bars (long dashed cyan line), long (short dashed red line) and short (dotted blue line). (b): bar fraction as a function of $\epsilon_{c}$ for long, short and long plus short barred galaxies. The vertical lines on both figures correspond to the expected value for unstable (solid red line) and self-gravitating (dashed black line) exponential disks.

and then an increase for galaxies with high spin. Error bars in the figure and subsequent ones denote the estimated $1 \sigma$ confidence intervals based on the bootstraping resampling method.

Dividing again the sample into long and short bars, we identify the first maximum with the increase of the fraction of long bars, while the second increase of the bar fraction is due to short bars. As we expected, the bar fraction is higher for galaxies with low spin parameter, although this is only for the case of long bars, the opposite is observed in the case of short bars, with the bar fraction increasing with increasing $\lambda_{d}$. If we look at the bar fraction as a function of the disk self-gravity parameter $\epsilon_{c}$ (Figure 2b), we see that only long bars fulfil the ENL stability criterion, while the frequency of short bars increases for galaxies that are expected to be stable against bar formation. It is worth noticing that the bar fraction drops for vanishing spin while still fulfilling the ENL stability criterion.

The difference of the $\epsilon_{c}$-distributions for the different subsamples shown on Figure 2a when compared with the $\lambda_{d}$ distributions on Figure 1 a, comes from the dependence on the disk mass fraction as estimated using equation 8. Typically, the long barred galaxies have 

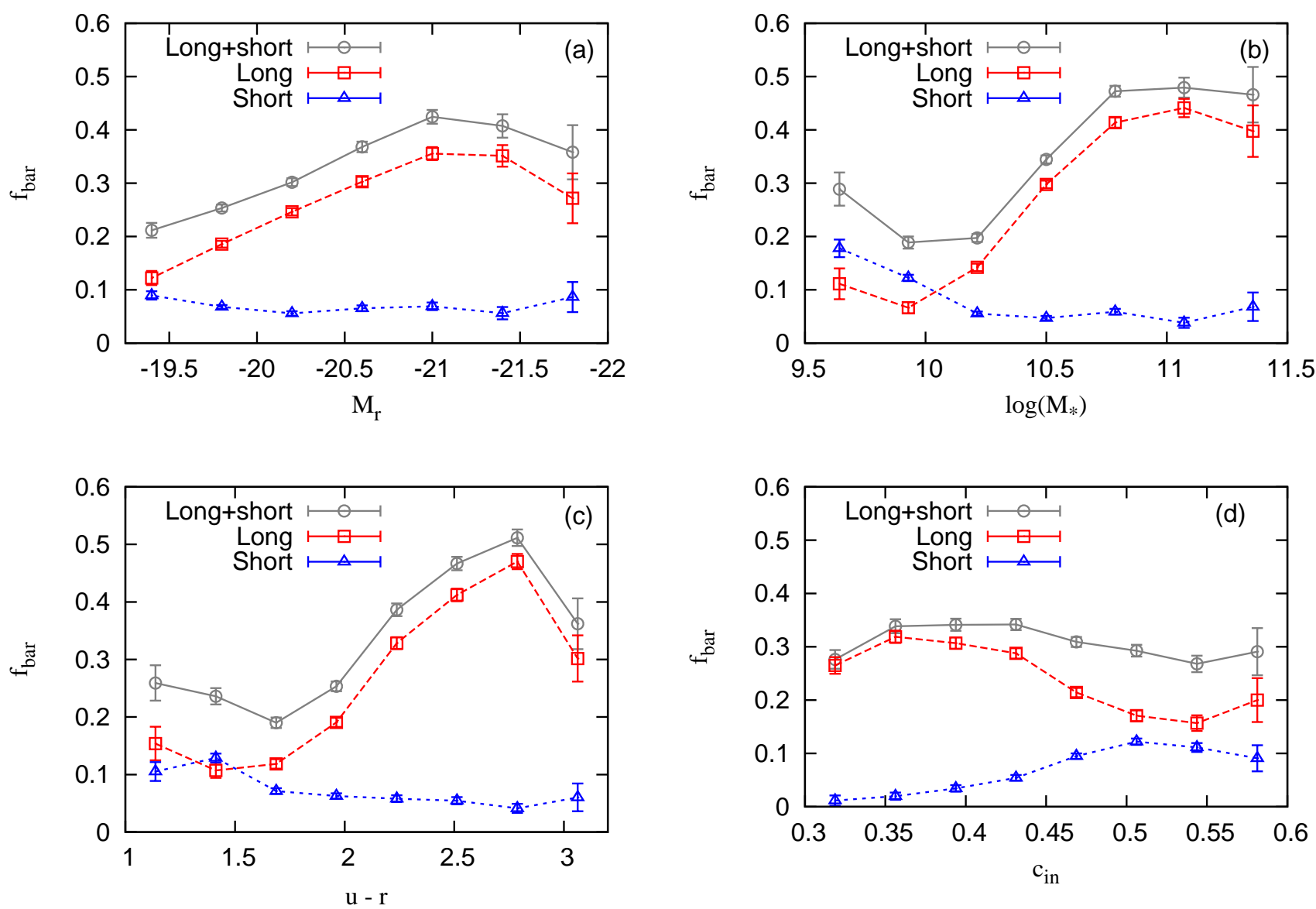

FIG. 3.- Dependence of bar fraction as a function of (a) $M_{r}$, (b) stellar mass, (c) $u-r$ color and (d) $c_{i n}$.

larger $f_{d}$ values than weak barred or unbarred galaxies. The case of using a constant dark matter fraction produces a qualitatively similar result on the dependence of the bar fraction on $\lambda_{d}$, only enhancing quantitatively the difference on the $\lambda_{d}$-distributions for the long and short barred galaxies. We present our results using this more conservative approach.

\subsection{Joint dependence of the bar fraction on the galactic spin and other galaxy properties}

Previous works have already shown the dependence of the occurrence of bars on different galaxy properties, such as luminosity, mass, color, concentration or gas content. On the other hand, we have also found correlations of the spin parameter with mass (Cervantes-Sodi et al. 2008), color (Hernandez \& Cervantes-Sodi 2006) and gas mass fraction (Cervantes-Sodi \& Hernandez 2009), among other parameters. For reference, Figure 3 shows the bar fraction as a function of absolute magnitude, stellar mass, color and concentration index, reproducing the findings by Lee+12 and Lee et al (2012b). Long bars are preferentially found in luminous, massive, red, highly concentrated galaxies (with low $i$-band inverse concentration index $\left.c_{i n}=R_{50} / R_{90}\right)$, while short barred galaxies are preferentially blue, less massive galaxies with low concentration indices. Within the magnitude limit of our sample, these distributions are in agreement with previous findings (i.e. Nair \& Abraham 2010; Méndez-Abreu et al. 2010).
To disentangle the dependence of the bar fraction on the spin and other crucial physical parameters, we look at the bar fraction in two parameter spaces involving the spin and other physical properties. We use a cubic B-spline kernel to get a smooth distribution of $f_{b a r}$, obtaining the ratio of the weighted number of barred galaxies to the total number of weighted galaxies using a fixed-size smoothing scale .

Figure 4 top panels presents the bar fraction in the $\lambda_{d}$ vs. $M_{r}$ space. The first thing to notice is that even at fixed $M_{r}$ there is a strong variation of $f_{b a r}$ as a function of the galactic spin for both cases, short as well as long bars. For the case of long bars(middle column), its clear that there is a maximum for bright galaxies with low spin parameters, although the bar fraction decreases for the brightest low spinning galaxies of the sample. It is worth noticing that the contours elongated in the $M_{r}$ direction shows that the $f_{b a r}$ for long bars shows a stronger dependence on $\lambda_{d}$ than on $M_{r}$. The case of short bars is less clear, at fixed $M_{r}$ there is a trend of increasing $f_{\text {bar }}$ with increasing $\lambda_{d}$, but no clear trend is found at fixed spin.

The brighter a galaxy is, the more massive it is, specially if we look at the absolute magnitude in red bands, that better traces the underlying stellar mass distribution and is less affected by current bursts of star formation. In Figure 4 second line we present the bar fraction in the $\lambda_{d}$ vs. $M_{*}$ space. $f_{b a r}$ for long bars shows a grad- 

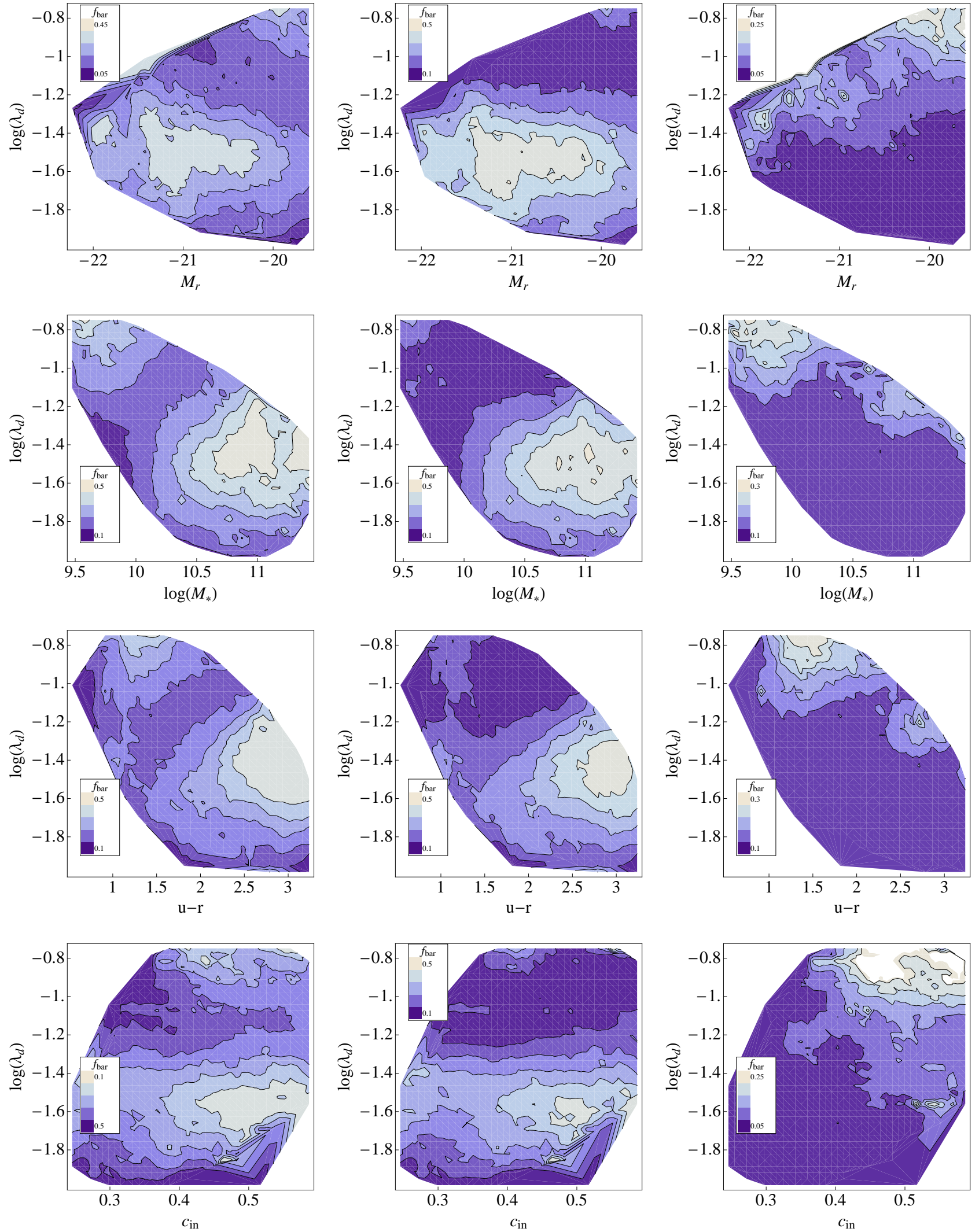

Fig. 4.- Bar fraction isocontours in the $\lambda_{d}$ vs. $M_{r}$ (first line), $M_{\text {stellar }}$ (second line), $u-r$ (third line) and $c_{i n}$ (forth line) space. Left column correspond to long plus short bars, middle to long bars and right to short bars. Each panel shows their corresponding $f_{\text {bar }}$ range and coding. 
ual increase for increasing stellar mass with a maximum at $11 \times 10^{10} M_{\odot}$ and then a slight decrease for the most massive galaxies in our sample. With massive galaxies having in general low spin parameter, this maximum correspond to low spinning galaxies, but at fixed stellar mass we can still notice a strong dependence on $\lambda_{d}$, and actually, for the most massive galaxies in our sample, those with moderate high $\lambda_{d}$ values are more prone to host long bars. The symmetry of the contours for the case of long bars tell us that the dependence on the spin parameter is as relevant as the dependence on stellar mass. Short bars are mostly present on low mass high spin galaxies

The third line of Figure 4 presents the bar fraction in the $\lambda_{d}$ vs. $u-r$ space. The double dependence on the chosen parameters is again noticeable. Although in our sample we can find blue galaxies with low spin parameter that fulfil the stability criterion for the formation of bars (equation 9), the long bar fraction of these galaxies is low when compared with red galaxies where $f_{\text {bar }}$ increases. Among red galaxies, systems with high spin present the highest bar fraction. This is also the case of short bars, but they reside in blue galaxies.

On the last line of Figure 4 we present the bar fraction in the $\lambda_{d}$ vs. $c_{i n}$ space. It is clear in this case that once we fix a specific value for $\lambda_{d}$, there is very little variation of the bar fraction varying the concentration index, with the exception of the less concentrated systems, specially for the case of long bars. Given this, we can attribute the dependence of $f_{b a r}$ on $c_{i n}$ directly to the spin of the galaxies.

\subsection{Dependence of the bar fraction on random motions}

As shown in Figure 1, the dependence of the bar fraction on the spin parameter is different for long and short bars.

For the case of galaxies with long bars, the bar fraction reaches its maximum at intermediate-low $\lambda_{d}$ values. Since it is the self-gravity of the disk which drives dynamical instabilities responsible for the formation of the bar, galaxies with low spin, being less dispersed, will be more prone to host a bar. Our finding of decreasing bar fraction with increasing $\lambda_{d}$ is in good agreement with the vanishing bar fraction on galaxies expected to be stable against bar formation as accounted by the ELN stability criterion.

It is important to point out that the maximum bar fraction is at low spin values, but as we approach zero, the bar fraction decreases dramatically. These galaxies are expected to be dynamically unstable and prone to the formation of bars as they are the most selfgravitating systems, but at the same time, as their spin value decreases, their rotational support is also diminished and random motions are expected to start dominating. Numerical studies (Athanassoula \& Sellwood 1986; Athanassoula 2008) show that disks that are expected to be unstable in terms of the ELN stability criterion become stable against bar formation due to their high-velocity dispersion, delaying the formation of a bar. Observational results confirm this hypothesis. Das et al. (2008) found on a sample of local galaxies that the bar strength and the normalized central velocity dispersion anti-correlate, suggesting that the bar weakens as the central component becomes kinematically hotter, and
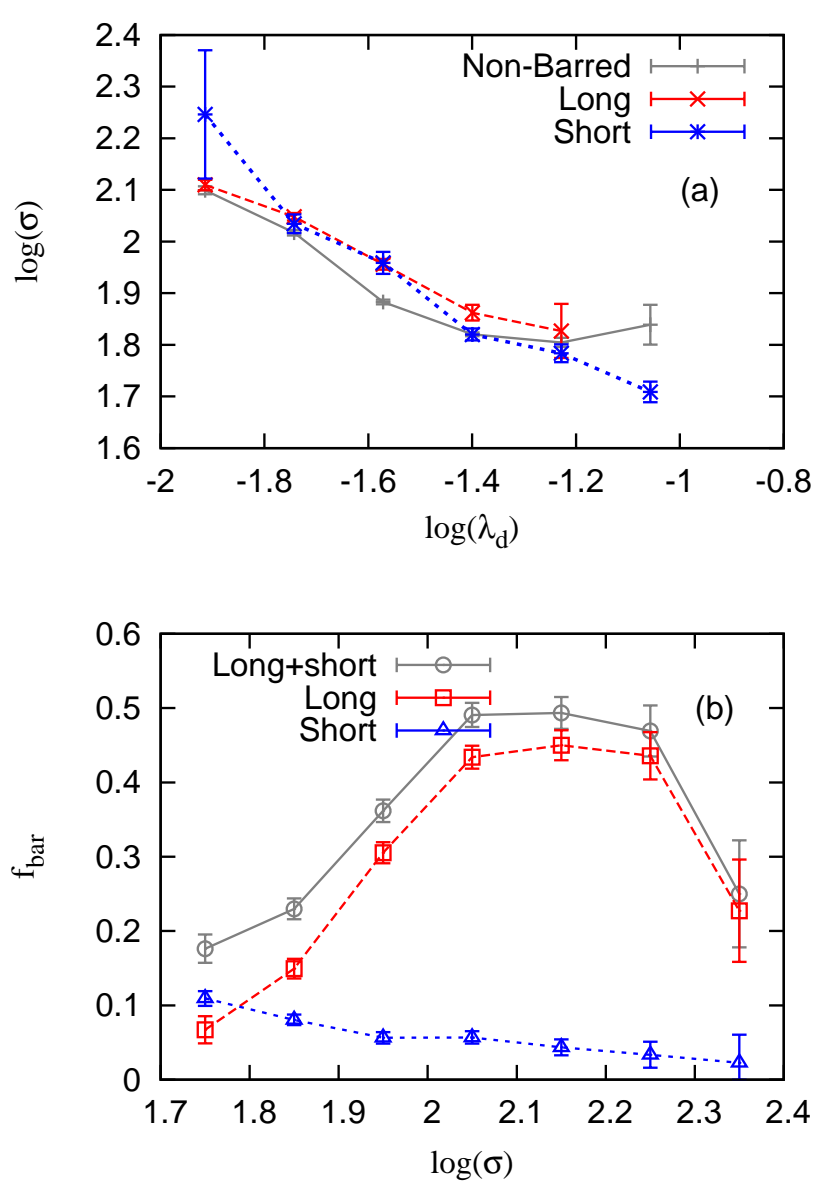

FIG. 5.- (a): Dependence of $\sigma$ on $\lambda_{d}$. (b): bar fraction as a function of $\sigma$.

more recently, Sheth et al. (2012) analysing a sample at higher redshift, found that bars where not present in dispersion-dominated disk galaxies. This gives a good explanation to the vanishing bar fraction as $\lambda_{d}$ approaches zero.

To give a further proof that this mechanism might be playing a role on the declining fraction of long bars in low spinning galaxies, we first investigate if the velocity dispersion shows any correlation with the spin parameter or the bar fraction. To get the minimum contribution from the rotation curve we limited our sample to those galaxies with $b / a>0.8$. Figure 5 a shows a clear anti-correlation of the velocity dispersion with the spin parameter that points in the direction we expect if we require low spinning galaxies to be supported by random motions. As already pointed out by Lee+12, the long bar fraction reaches its maximum at intermediate velocity dispersion values in the range $125-175 \mathrm{~km} \mathrm{~s}^{-1}$, as we notice in Figure 5 b. Lee +12 also concluded that the decline of the bar fraction for $\sigma>175 \mathrm{~km} \mathrm{~s}^{-1}$ could be due to the disruption of bars in galaxies with centers dynamically too hot, or that bar formation was ultimately suppressed on such systems (see Athanassoula et al. 2013).

The corresponding two dimensional maps for the bar fraction in the $\log (\sigma)$ vs. $\log \left(\lambda_{d}\right)$ space are shown in Figure 6. In agreement with what we expected, we can first notice the anti-correlation between the two physical parameters, and for the case of long bars, a clear decrease 

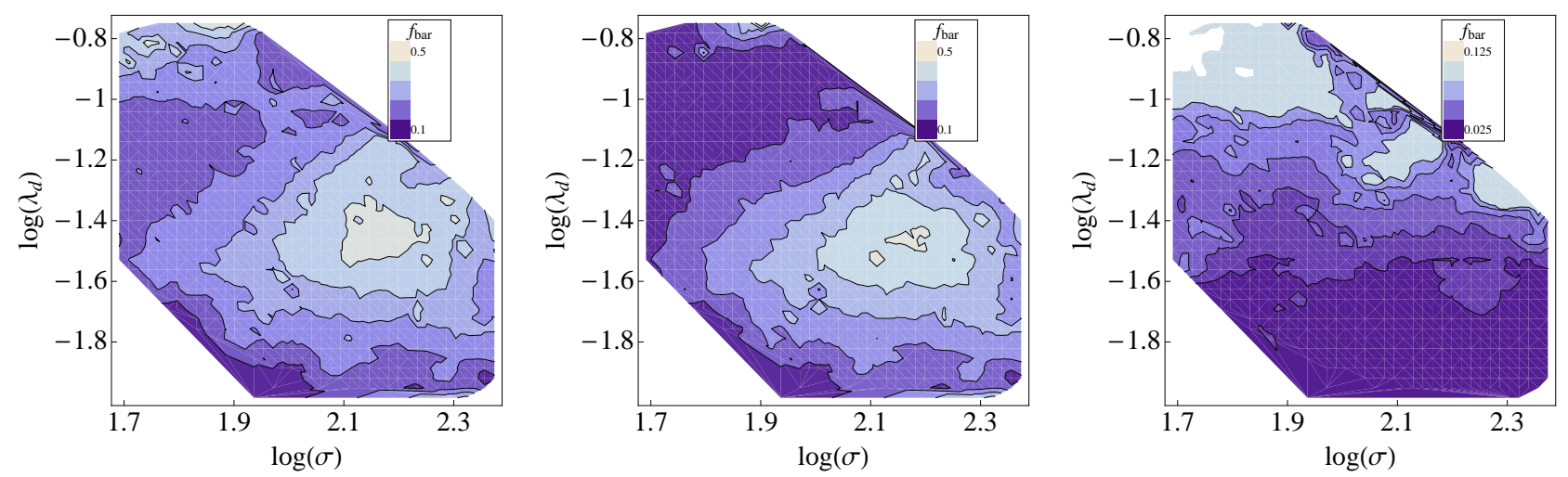

FIG. 6. - Bar fraction isocontours in the $\lambda_{d}$ vs. $\sigma$ space. Left column correspond to long plus short bars, middle to long bars and right to short bars. Each panel shows their corresponding $f_{b a r}$ range and coding.

of the bar fraction, even for low spinning systems, with $\sigma>160 \mathrm{~km} \mathrm{~s}^{-1}$. Although this cannot be regarded as a prove for our hypothesis, given that our $\lambda_{d}$ estimation comes from invoking a TF relation, and not from a pure kinematic study, this result can be explained as a result of two competing factors, the self-gravity of the disk that enhances the formation of bars, and the support by random motions that works in the opposite direction, preventing bar instabilities.

\section{DISCUSSION}

Galaxies hosting a short bar have typically higher spin than those hosting long bars or non-barred galaxies, with the bar fraction increasing with increasing $\lambda_{d}$. The presence of these short bars on high $\lambda_{d}$ systems confront the ELN stability criterion as shown in Figure 2 2 , where the bar fraction increases even for galaxies with $\epsilon_{c}>1.1$. As pointed out by Sellwood \& Moore (1999), while the normal bar instability gives a natural explanation for the presence of strong bars, there is no alternative mechanism to explain the existence of weak bars. Erwin (2005) presents a careful and extensive comparison of bar lengths between observational and numerical results and concludes that the simulations compared with his results tend to produce long bars, and except two early simulations (Pfenniger \& Friedli 1991; Combes \& Elmegreen 1993), no N-body bars are as small as typical Sc-Sd bars, that are expected to be galaxies with a high spin parameter.

An extreme case of high spinning systems is low surface brightness galaxies (LSBs). Simulations with $\lambda_{d}>0.04$ are able to reproduce some of the physical characteristics of these galaxies such as their surface density profiles and colors (e. g. Jimenez et al. 1998; Kim \& Lee 2012), and while they are expected to be stable to bar formation due to the low self-gravity of their disks and be dark matter dominated at all radii, some simulations are able to produce bars that display a mild oval distortion (Mihos, McGaugh \& de Block 1997), with short-lived episodes that finally evolve to a bulge-like structure (Mayer \& Wadsley 2004).

The fact that bars appear short on high spinning galaxies could be due to the low efficiency of their disks as angular momentum sinks. The disperse disks of these systems are unable to carry away the necessary amount of angular momentum from the bar in order to grow to the extent of the typical bars of low spinning galaxies.

Recently, Scannapieco \& Athanassoula (2012) studied the properties of bars formed in fully cosmological hydrodynamical simulations of Milky Way-mass galaxies and found that the longest bar is formed in a bulge-disk-halo system with the lowest spin parameter, and the shortest is found in a galaxy with a disk but not significant bulge and the highest spin parameter. Previous studies have shown similar results (Athanassoula \& Misiriotis 2002; Ahtanassoula 2003), stronger bars residing in galaxies with prominent bulges. Hernandez \& CervantesSodi (2006) noticed that galaxies with the largest bulgeto-disk ratio are those with low spin parameter, a result that fits in this picture of galaxies with low values of $\lambda_{d}$ presenting longer bars that galaxies with high spin.

The competing effects of self-gravity in development and growth of the bar instability and the suppression through random motions in dispersion dominated systems helps to explain the dependence of the bar fraction on the galactic spin. Given the clear trend for mean $\lambda_{d}$ values increasing in going towards later Hubble types (Cervantes-Sodi \& Hernandez 2009 and references therein), our results are in good agreement with previous studies regarding the bar fraction as a function of morphological type, in the sense that bars in early-type, low spinning galaxies, are longer than those in late-type, high spinning ones (e.g. Erwin 2005).

An additional factor that might be playing an important role is the content of cold gas. Galaxies with high spin have typically late-type morphology, with blue colors and higher gas mass fractions. In these systems the ELN stability criterion might not apply as pointed out by Christodoulou et al. (1995), given the big influence the gas has in the formation and development of stellar bars. The threshold value found in simulations varies depending on the amount of gas and the type of cooling that is implemented (Mayer \& Wadsley 2004), but in general the gas component severely limits the bar growth and evolution (Villa-Vargas et al. 2010), usually producing weak, short-lived bars. Previously, Shlosman \& Noguchi 
(1993) studied the effect of gas on the global stability of a galactic disk embedded in a live halo, and they found two different regimes; in a low gas surface density disk, the radial redistribution of the gas depends solely on the stability of the stellar disk, while for high gas surface density, the gas develops inhomogeneities that heat up the stellar component that increases the stability of the system preventing the growth of a bar. This result fits well with our finding, where long bars are hosted by luminous, massive, red galaxies that typically present low gas content, while short bars are found in low luminosity, low mass, blue galaxies which are usually gas rich.

On a recent study by Athanassoula et al. (2013), the authors follow the formation and evolution of bars in Nbody simulations of disk galaxies with gas, where the gas component is modeled as a multiphase medium, including star formation, feedback and cooling. The study shows that in gas-rich simulations, the disk stays axisymmetric longer than in gas-poor ones, and once the bar forms, it grows at a much slower rate. This explains why fully grown bars are in place earlier in massive red disks than in blue spirals. In a subsequent study, we will investigate the combined effect of gas content and spin on the bar properties of disk galaxies.

\section{CONCLUSIONS}

In summary, we report a strong dependence of the bar fraction on the galactic spin of disk galaxies. This dependence on $\lambda_{d}$ is different for long and short bars. Long bars are preferentially found in galaxies with low to intermediate $\lambda_{d}$ that are more prone to develop bar instabilities due to their self-gravitation. These galaxies are typically massive, luminous, red, gas poor systems, when compared with galaxies hosting short bars. Instead, short bars are mostly found in high spinning galaxies, that at the same time are typically low mass, faint galaxies, with blue colors and rich in cold gas.

The rise and fall of the bar fraction for the case of long bars as a function of $\lambda_{d}$ can be explained as the result of two competing factors, the self-gravity of the systems and the support by random motions. At high $\lambda_{d}$ values, the decline of the bar fraction is due to the lack of selfgravity, being the disk more sparse the global instabilities are suppressed or damped. At low $\lambda_{d}$ values, the support of the system by random motions instead of ordinate rotation becomes predominant; that also prevents the formation and growth of bars.

Our finding of short bars being hosted by high spinning galaxies is in good agreement with previous observational and theoretical studies, considering that these systems are preponderantly blue, gas rich galaxies; where the sparsity of the disk material and the large fraction of gas play an important role on restricting the formation/growth of the bar.

The authors thank the referee for his/her constructive comments that helped to improve the manuscript. BC-S acknowledge E. Athanassoula and Issac Shlosman for helpful comments. This work is supported by NSFC (no. 11173045 and 11233005), Shanghai Pujiang Programme (no. 11PJ1411600) and the CAS/SAFEA International Partnership Programme for Creative Research Teams (KJCX2-YW-T23)

Funding for the SDSS and SDSS-II has been provided by the Alfred P. Sloan Foundation, the Participating Institutions, the National Science Foundation, the U.S. Department of Energy, the National Aeronautics and Space Administration, the Japanese Monbukagakusho, the Max Planck Society, and the Higher Education Funding Council for England. The SDSS Web Site is http://www.sdss.org/. The SDSS is managed by the Astrophysical Research Consortium for the Participating Institutions. The Participating Institutions are the American Museum of Natural History, Astrophysical Institute Potsdam, University of Basel, University of Cambridge, Case Western Reserve University, University of Chicago, Drexel University, Fermilab, the Institute for Advanced Study, the Japan Participation Group, Johns Hopkins University, the Joint Institute for Nuclear Astrophysics, the Kavli Institute for Particle Astrophysics and Cosmology, the Korean Scientist Group, the Chinese Academy of Sciences (LAMOST), Los Alamos National Laboratory, the Max-Planck-Institute for Astronomy (MPIA), the Max-Planck-Institute for Astrophysics (MPA), New Mexico State University, Ohio State University, University of Pittsburgh, University of Portsmouth, Princeton University, the United States Naval Observatory, and the University of Washington.

\section{REFERENCES}

Abazajian, K. N., Adelman-McCarthy, J. K., Agüeros, M. A., et al. 2009, ApJS, 182,543

Aguerri, J. A. L., Méndez-Abreu, J., \& Corsini, E. M. 2009 , A\&A, 495, 491

Abraham, R. G., Merrifield, M. R., Ellis, R. S., Tanvir, N. R., \& Brinchmann, J. 1999, MNRAS, 308, 569

Athanassoula, E. 2002, ApJ, 569, L83

Athanassoula, E. 2003, MNRAS, 341, 1179

Athanassoula, E. 2008, MNRAS, 390, L69

Athanassoula, E., Lambert, J. C., \& Dehnen, W. 2005, MNRAS, 363,496

Athanassoula, E., Machado, R. E. G., \& Rodionov, S. A. 2013, MNRAS, 429, 1949

Athanassoula, E., \& Misiriotis, A. 2002, MNRAS, 330, 35

Athanassoula, E., \& Sellwood, J. A. 1986, MNRAS, 221, 213

Barazza, F. D., Jogee, S., \& Marinova, I. 2008, ApJ, 675, 1194

Bournaud, F., \& Combes, F. 2002, A\&A, 392, 83

Bournaud, F., Combes, F., \& Semelin, B. 2005, MNRAS, 364, L18

Bruzual, G., \& Charlot, S. 2003, MNRAS, 344, 1000

Bullock, J. S., Dekel, A., Kolatt, T. S., Kravtsov, A. V., Klypin, A. A., Porciani, C., \& Primack, J. R. 2001, ApJ, 555, 240

Buta, R., \& Combes, F. 1996, Fund. Cosmic Phys., 75, 95
Buta, R., Laurikainen, E., Salo, H., \& Knapen J. H. 2010, ApJ, 721,259

Cervantes-Sodi, B., \& Hernandez, X. 2009, RevMexA\&A, 45, 75

Cervantes-Sodi, B., Hernandez, X., Hwang, H. S., Park, C., \& Le Borgne, D. 2012, MNRAS, 426, 1606

Cervantes-Sodi, B., Hernandez, X., Park, C., \& Choi, Y.-Y. 2011, ApJ, 735, L25

Cervantes-Sodi, B., Hernandez, X., Park, C., \& Kim, J. 2008, MNRAS, 388, 863

Choi, Y.-Y., Han, D., \& Kim, S. S. 2010, J. Korean Astron. Soc., 43, 191

Christodoulou, D.M., Shlosman, I., \& Tohline, J.E. 1995, ApJ, 443,551

Coelho, P., \& Gadotti, D. A. 2011, ApJ, 743, L13

Combes, F. 2000, in Dynamics of Galaxies from the Early

Universe to the Present, ed. F. Combes, G. A. Mamon, \& V. Charmandaris, ASP Conf. Ser., 197, 15

Combes, F., \& Elmegreen, B. G. 1993, A\&A, 271, 391

Courteau, S., Andersen, D. R., Bershady, M. A., MacArthur, L. A., Rix., H.-W. 2003, ApJ, 594, 208

Das, M., Laurikainen, E., Salo, H., \& Buta, R. 2008, Ap\&SS, 317, 163 
Debattista, V. P. \& Sellwood, J. A. 2000, ApJ, 543, 704

Debattista, V. P., Mayer, L., Carollo, C. M., et al. 2006, ApJ, 645, 209

Efstathiou, G., Lake, G., \& Negroponte, J. 1982, MNRAS, 199, 1069

Ellison, S. L., Nair, P., Patton, D. R., et al. 2011, MNRAS, 416, 2182

Elmegreen, B. G., \& Elmegreen, D. M. 1985, ApJ, 288, 438

Elmegreen, B. G., \& Elmegreen, D. M. 1985, ApJ, 342, 677

Elmegreen, B. G., Elmegreen, D. M., \& Hirst, A. C. 2004, ApJ, 612,191

Erwin, P. 2005, MNRAS, 364, 283

Foyle, K., Courteau, S., \& Thacker, R. J. 2008, MNRAS, 386 , 1821

Friedli, D., \& Benz, W. 1993, A\&A, 268, 65

Friedli, D., Benz, W., \& Kennicutt, R. 1994, ApJ, 430, L105

Gadotti, D. A. 2011, MNRAS, 415, 3308

Gnedin, O. Y., Weinberg, D. H., Pizagno, J., Prada, F., \& Rix, H.-W. 2007, ApJ,671, 1115

Gogarten, S. M., Dalcanton, J. J., Williams, B. F., et al. 2010, ApJ, 712, 858

Governato, F., Brook, C. B., \& Brooks, A. M. et al. 2009, MNRAS, 398, 312

Hasan, H., \& Norman, C. A. 1990, ApJ, 361, 69

Hernandez, X., \& Cervantes-Sodi, B. 2006, MNRAS, 368, 351

Hernandez, X., Park, C., Cervantes-Sodi, B., \& Choi, Y.-Y. 2007, MNRAS, 375, 163

Hohl, F. 1971, ApJ, 168, 343

Hoyle, B., et al. 2011, MNRAS, 415, 3627

Huang, J. H., Gu, Q. S., Su, H. J., et al. 1996, A\&A, 313, 13

Hummel, E., van der Hulst, J. M., Kennicutt, R. C., \& Keel, W. C. 1990, A\&\& $, 236,333$

Hwang, J.-S., Park, C., \& Choi, J.-H. 2013, JKAS, 46, 1

Jimenez, R., Padoan, P., Matteucci, F., \& Heavens, A. F. 1998, MNRAS, 299, 123

Jogee, S., et al. 2004, ApJ, 615, L105

Kauffmann, G., Heckman, T. M., White, S. D. M., et al. 2003, MNRAS, 341, 33

Kim, J.-H., \& Lee, J. 2013, MNRAS, 432, 1701

Knapen, J. H., Shlosman, I., \& Peletier, R. F. 2000, ApJ, 529, 93

Kormendy, J., \& Kennicutt, R. C., Jr. 2004, ARA\&A, 42, 603

Laine, S., Shlosman, I., Knapen, J. H., \& Peletier, R. F. 2002, ApJ, 567, 97

Lauberts, A. 1982, The ESO/Uppsala Survey of the ESO(B) Atlas, European Southern Observatory

Laurikainen, E., Salo., H., \& Buta, R., 2004, ApJ, 607, 103

Laurikainen, E., et al. 2007, MNRAS, 381, 401

Lee, G.-H., Park, C., Lee, M. G., \& Choi, Y.-Y. 2012a, ApJ, 745, 125

Lee, G.-H., Woo, J.-H., Lee, M. G., Hwang, H. S., Lee, J. C., Sohn, J., \& Lee, J. H. 2012b, ApJ, 750, 141

Lindblad, P. O. 1960, Stockholms observatoriums annaler, bd. 21 , 4

Marinova, I., \& Jogee, S. 2007, ApJ, 659, 1176
Márquez, I., Durret, F., Masegosa, J., et al. 2000, A\&A, 360, 431 Martinez-Valpuesta, I., Shlosman, I., \& Heller, C. 2006, ApJ, 637, 214

Martini, P., \& Pogge, R. W. 1999, AJ, 118, 2646

Masters, K. L., et al. 2011, MNRAS, 411, 2026

Mayer, L., \& Wadsley, J. 2004, MNRAS, 347, 277

Méndez-Abreu, J., Sánchez-Janssen, R., \& Aguerri, J. A. L. 2010 ApJ, 711, L61

Menéndez-Delmestre, K., Sheth, K., Schinnerer, E., Jarrett, T.

H., \& Scoville, N. Z. 2007, ApJ, 657, 790

Mihos, J. C., McGaugh, S. S., \& de Blok, W. J. G. 1997, ApJ, 477, L79

Mo, H. J., Mao, S., \& White, S. D. M. 1998, MNRAS, 295, 319

Moles, M., Marquez, I., \& Perez, E., 1995, ApJ, 438, 604

Mulchaey, J. S. \& Regan, M. W. 1997, ApJ, 482, L135

Muoz-Mateos, J. C., Boissier, S., Gil de Paz, A., Zamorano, J., Kennicutt, R. C., Jr., Moustakas, J., Prantzos, N., \& Gallego, J. 2011, ApJ, 731, 10

Nair, P. B., \& Abraham, R. G. 2010, ApJ, 714, L260

Norman, C. A., Sellwood, J. A., \& Hasan, H. 1996, ApJ, 462, 114

Oh, S., Oh, K., \& Yi, S. K. 2012, ApJ, 198, 4

Okamoto, T. 2012, MNRAS, doi: $10.1093 / \mathrm{mnras} / \mathrm{sts} 067$

Park, C., \& Choi, Y.-Y. 2005, ApJ, 635, L29

Peebles, P. J. E 1971, A\&A, 11, 377

Pfenniger, D., \& Friedli, D. 1991, A\&A, 252, 75

Pizagno, J., Prada, F., Weinberg, D. H., et al. 2007, AJ, 134, 945

Puech, M., Hammer, F., Lehnert, M. D., \& Flores, H. 2007,

A\&A, 466, 83

Sakamoto, K., Okumura, S. K., Ishizuki, S., \& Scoville, N. Z. 1999, ApJ, 525, 691

Scannapieco, C., \& Athanassoula, E. 2012, MNRAS, 425, L10

Schwartz, M. P. 1981, ApJ, 247, 77

Sellwood, J. A., \& Moore, E. M. 1999, ApJ, 510, 125

Shaw, L. D., Welle,r J., Ostriker, J. P., \& Bode, P. 2006, ApJ, 646,815

Shen, J., \& Sellwood J. A. 2004, ApJ, 604, 614

Sheth, K., Elmegreen, D. M., Elmegreen, B. G., et al. 2008, ApJ, 675,1141

Sheth, K., Vogel, S. N., Regan, M. W., Thornley, M. D., \&

Teuben, P. J. 2005, ApJ, 632, 217

Sheth, K., Melbourne, J., Elmegreen, D. M., Elmegreen, B. G. Athanassoula, E., Abraham, R. G., \& Weiner, B. J. 2012, ApJ, 758,136

Shlosman, I., Frank, J., \& Begelman, M. C. 1989, Nature, 338, 45 Shlosman, I., \& Noguchi, M. 1993, ApJ, 414, 474

Shlosman, I., Peletier, R. F., \& Knapen, J. H. 2000, ApJ, 535, L83

Syer, D., Mao, S., \& Mo, H. J. 1999, MNRAS, 305, 357

Toomre, A. 1960, ApJ, 158, 899

van den Bergh, S., Abraham, R. G., Whyte, L. F., Merrifield, M. R., Eskridge, P. B., Frogel, J. A., \& Pogge, R. 2002, AJ, 123 2913

Villa-Vargas, J., Shlosman, I., \& Heller, C. 2010, ApJ, 719, 1470 Weinberg, M. D. 1985, MNRAS, 213, 451 